\title{
The relationship between serum cobalamin, folic acid, and homocysteine and the risk of post-cardiac surgery delirium
}

This article was published in the following Dove Press journal:

Neuropsychiatric Disease and Treatment

\author{
Maryam Vahdat Shariatpanahi' \\ Aynaz Velayati ${ }^{2}$ \\ Seyed Ali Jamalian ${ }^{3}$ \\ Mehdi Babevaynejad ${ }^{4}$ \\ Zahra Vahdat Shariatpanahi ${ }^{2}$ \\ 'Department of Psychiatry, Faculty of \\ Medicine, Tehran Medical Sciences, Islamic \\ Azad University, Tehran, Iran; ${ }^{2}$ National \\ Nutrition and Food Technology Research \\ Institute, Faculty of Nutrition and Food \\ Technology, Shahid Beheshti University of \\ Medical Sciences, Tehran, Iran; \\ ${ }^{3}$ Department of Cardiology, Shahid \\ Lavasani Hospital, Islamic Azad University, \\ Tehran, Iran; ${ }^{4}$ Department of Cardiac \\ Surgery, Shahid Lavasani Hospital, Islamic \\ Azad University, Tehran, Iran
}

\begin{abstract}
Purpose: It has been reported that cobalamin and folate deficiency is related to delirium in persons with dementia. We evaluated the association of admission serum levels of cobalamin, folic acid, and homocysteine with the occurrence of acute delirium after coronary artery bypass surgery.
\end{abstract}

Methods: In this prospective cohort study, serum levels of cobalamin, folic acid, and homocysteine were measured for 296 patients upon admission. Delirium was defined by the confusion assessment method for the intensive care unit.

Results: Postoperative delirium was detected in $23 \%(n=68)$ of patients. Cobalamin deficiency, folate deficiency, and hyperhomocysteinemia were observed in $29 \%(n=86), 6 \%(n=18)$, and $68 \%$ $(n=200)$ of patients, respectively. The mean \pm SD serum levels of folic acid were $10.77 \pm 5.39 \mathrm{ng} / \mathrm{mL}$ and $12.86 \pm 6.51 \mathrm{ng} / \mathrm{mL}$ in delirium and non-delirium patients, respectively $(P=0.008)$. The median (interquartile range [IQR]) serum levels of cobalamin were 280 (216-351) and 247 (195.5-336) in delirium and non-delirium patients, respectively $(P=0.09)$. The median (IQR) serum levels of homocysteine were $18.5(14.5-22.1) \mu \mathrm{mol} / \mathrm{L}$ and 17.33 (14.2-23.2) $\mu \mathrm{mol} / \mathrm{L}$ in delirium and nondelirium patients, respectively $(P=0.94)$. Multivariate regression analysis adjusted by other risk factors indicated that serum homocysteine, folate, and cobalamin levels had no association with the occurrence of delirium.

Conclusion: There was no relationship between the preoperative levels of cobalamin, folate, and homocysteine, and acute occurrence of delirium observed after cardiac surgery.

Keywords: vitamin B12, CAM-ICU, cardiopulmonary bypass, intensive care unit

\section{Introduction}

Delirium is a disorder defined as an acute disturbance in attention, memory, cognition, and alteration of consciousness. ${ }^{1,2}$ Postoperative delirium (POD) is a kind of delirium that develops immediately after the operation and takes a long time to go away from hours to days. ${ }^{3}$ Its prevalence after the cardiac operation has been reported to be $8-54.9 \%{ }^{4}$ Delirium has been categorized into three subtypes: 1) hypoactive delirium, characterized by apathy, withdrawal, lethargy, and decreased responsiveness, 2) hyperactive delirium which is characterized by turbulence, restlessness, and agitation, and 3) mixed delirium which vacillates between hypoactive and hyperactive types. ${ }^{4}$ POD is strongly associated with mortality, long-term cognitive disorder, higher duration of mechanical ventilation, nursing costs, hospital readmission levels, and probability of transfer to mental institutions. ${ }^{1,5,6}$ In order to reduce the
Correspondence: Zahra Vahdat Shariatpanahi

3, Baran, West Arghavan, Farahzadi Blvd., Shahrak Qods, PO Box: 19395-474I,

Tehran, Zip Code: 1981619573, Iran

Tel +98 2122357483 ext 4

Fax +98 2122376467

Email nutritiondata@yahoo.com 
incidence of POD, it is important to recognize its predisposing risk factors, such as older age, coexisting medical conditions, electrolyte disturbances, anemia, frequent alcohol consumption, impaired hearing and vision, preoperative psychiatric problems, preoperative neurological diseases, and prolonged ICU stay. Among these factors, the role of nutritional deficiencies has been less evaluated.

Cobalamin (vitamin B12) deficiency is one of the most prevalent micronutrient deficiencies around the world, ${ }^{7}$ and is reported to be present in $25 \%$ of the patients with cardiovascular diseases. ${ }^{8}$ Among the complications of cobalamin deficiency are neurological symptoms, depression, ${ }^{9}$ dementia, ${ }^{10}$ catatonia, ${ }^{11}$ schizophrenia, ${ }^{12}$ and psychosis. ${ }^{13}$ A recent retrospective study showed that cobalamin deficiency is a risk factor for delirium following cardiac surgery. ${ }^{14}$ Similar to B12 deficiency, folate deficiency can also lead to neurological disorders, cognitive dysfunction, and dementia. ${ }^{15}$ Folate deficiency is reported to be present in $29 \%$ of the patients with cardiovascular diseases. ${ }^{15}$ Both B12 and folate deficiency entail hyper-homocysteinaemia (HHC), which is also a risk factor for neurological disorders. $^{16}$ In addition, preoperative HHC is associated with worse postoperative outcomes. ${ }^{17}$

To the best of our knowledge, the association between the serum levels of folate and homocysteine and POD has not been specified in cardiac surgery. Coronary artery bypass grafting (CABG) surgery is a common surgery and is associated with high incidence of POD, hence the fact that we evaluated the relationship between preoperative serum cobalamin, folic acid, and homocysteine levels with post-CABG surgery delirium in a cardiac surgery ICU.

\section{Materials and methods Study design and participants}

In this prospective cohort study, data were collected between May 2017 and May 2018 from a postcardiac intensive care unit in a university-affiliated hospital. Patients' $\geq 18$ years old and admitted in hospital for CABG surgery were included in our study. Exclusion criteria were preoperatively diagnosed delirium, mental disease, dementia, and death in less than $24 \mathrm{hrs}$ in ICU. Eligible patients or their legal representatives signed informed consent. The study was approved by the Shahid Beheshti University of Medical Sciences Ethics committee, hence performed in accordance with the ethical standards laid down in the 1964 Declaration of Helsinki and its later amendments.

\section{Measurements}

A day before CABG surgery, venous blood samples were obtained from all patients for the measurement of serum cobalamin, folate, and homocysteine. Homocysteine levels were measured using enzyme immunoassay (EIA) method (Axis-Shield Diagnostics, Scotland). Hyperhomocysteinemia was classified into moderate $(15-30 \mu \mathrm{mol} / \mathrm{L})$, intermediate $(30-100 \mu \mathrm{mol} / \mathrm{L})$, and severe (greater than $100 \mu \mathrm{mol} / \mathrm{L}$ ) types based on the level of Homocysteine. ${ }^{18}$ Serum folate was determined via EIA method (Roche Diagnostic, USA) and values less than $3 \mathrm{ng} / \mathrm{mL}$ were considered as indicative of folic acid deficiency. ${ }^{19}$ Serum cobalamin was estimated utilizing the EIA method (Roche Diagnostic, USA), and values less than $200 \mathrm{pg} / \mathrm{mL}$ were considered as indicative of cobalamin deficiency, and amounts between 200 and $300 \mathrm{pg} / \mathrm{mL}$ were considered as borderline. ${ }^{19}$

\section{Delirium assessment}

After surgery, patients were transferred to the cardiac surgery ICU with standard post-operative care and were monitored for the occurrence of delirium. Delirium was diagnosed according to the confusion method for the ICU (CAM-ICU), which was performed for each patient after assessing the patients' consciousness with the Richmond Agitation-Sedation Scale (RASS). If the RASS score was -3 to +4 , CAM-ICU was performed later. Delirium was diagnosed if the following criteria were met: an acute onset of changes or fluctuations in the course of mental status over the last $24 \mathrm{hrs}$, inattention, disorganized thought, or an altered level of consciousness. Delirium was assessed by a nurse trained in working CAM-ICU, and the results were confirmed by ICU physicians involved in the patients' care.

\section{Data collection}

The cofounder variables potentially related to the development of post-CABG surgery delirium were recorded for further analysis. These were age, sex, education level (illiterate, elementary or middle school degree, high school degree, college education), smoking (tobacco use at least three months before surgery), alcohol use (three months before surgery), impaired hearing or vision, hypertension $(\geq 140 / 90 \mathrm{mmHg}$ ), diabetes mellitus, renal insufficiency, hemoglobin level $<12 \mathrm{mg} / \mathrm{dL}$ in men and $<11 \mathrm{mg} / \mathrm{dL}$ in women, serum electrolyte disturbances, poor quality of sleep, early mobilization, and acute infection. Mobilization was assessed as good, average, and bad according to the 
willingness of the patient for postoperative early mobilization. Sleep quality was assessed by self-report by the patient.

\section{Statistical analyses}

The collected data were statistically analyzed with SPSS software (Version 24.0. Armonk, NY: IBM Corp). $P$-values less than 0.05 were considered significant. Descriptive statistics were used to characterize and summarize the study population. Data were shown as frequencies and percentages or median with interquartile range (IQR), as most of the continuous variables were highly skewed and not normally distributed. Chi-square test was used to check the differences in the distribution of categorical variables. In addition, the Mann-Whitney test was used to assess the differences in the distribution of continuous variables between delirium and non-delirium patients. Logistic regression analysis was used to assess the relationship between serum cobalamin, folate, homocysteine, and occurrence of post CABG delirium.

\section{Results}

A total of 350 patients participated in the study. Fifty-four were excluded due to dissatisfaction with the operation, death before the second admission day in ICU and missing data. Therefore, 296 patients (64 females (22\%) and 232 males $(78 \%)$ ) were included in the study (Figure 1). Table 1

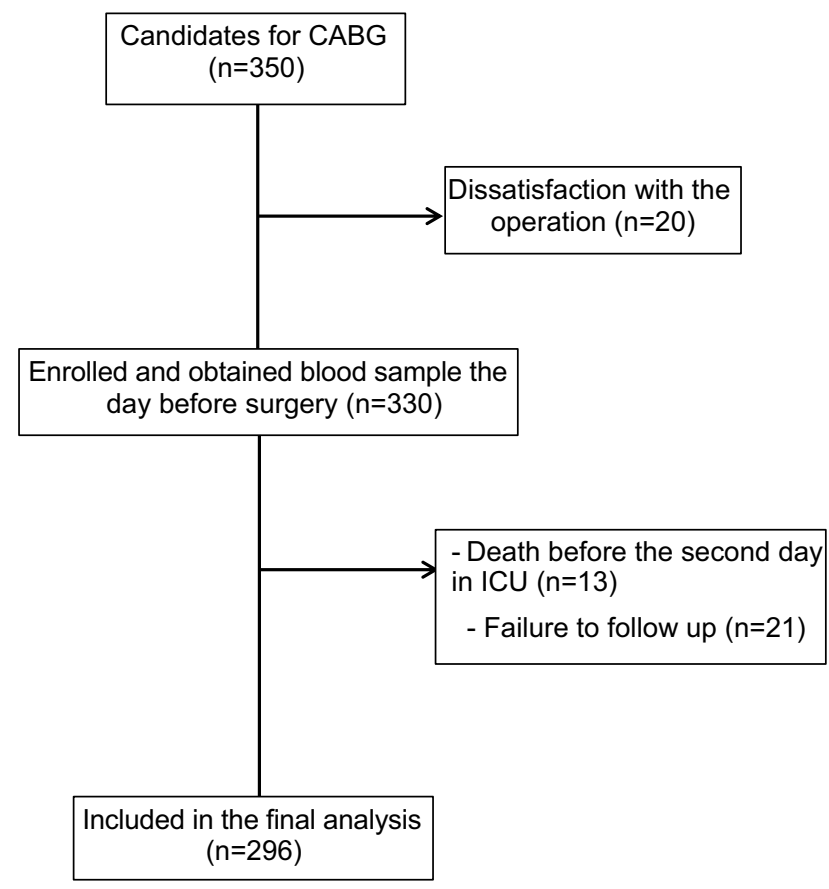

Figure I Study flowchart.

Abbreviation: CABG, coronary artery bypass grafting. presents the baseline characteristics of patients based on delirium and no delirium. The median age of participants was 62 years (IQR, 54-66). Delirium was detected in 23\% of the patients $(n=68)$. There was no significant difference with regard to sex, smoking, addiction, and duration of operation between the two groups. Cardiopulmonary bypass was performed for all patients. Type of operation was isolated CABG surgery with single-vessel disease $(7 \%, n=20)$, isolated $\mathrm{CABG}$ surgery with two-vessel disease $(19 \%$, $\mathrm{n}=56$ ), isolated CABG surgery with three-vessel disease $(70 \%, \mathrm{n}=208)$, and valvular plus CABG surgery $(4 \%$, $\mathrm{n}=12$ ), with no significant difference between the two groups. According to NYHA classification, 80\% $(n=236)$ of the total population were in class II, $13 \%(n=38)$ were in class III, and $7 \%(n=22)$ belonged in class IV, with no significant difference between the two groups. Median length of stay in the ICU and hospital was significantly higher in the delirium group.

\section{Serum level of cobalamin and outcomes}

The median (IQR) serum level of cobalamin was 256.5 (IQR, 197.75-340) $\mathrm{pg} / \mathrm{mL}$ in the total population. Cobalamin deficiency with a serum level of lower than $200 \mathrm{pg} / \mathrm{mL}(<148$ $\mathrm{pmol} / \mathrm{L})$ was observed in $29 \%(\mathrm{n}=86)$ of patients, and $35 \%$ $(n=104)$ had borderline levels. The median serum level of cobalamin was 280 (IQR, 216-351) pg/mL in the delirium group and 247 (IQR, 195.5-336) pg/mL in the non-delirium group ( $P=0.09$ ). The median length of stay in the ICU and hospital was 2 days vs 3 days and 5 days vs 6 days in patients with and without cobalamin deficiency, respectively; their differences were not significant $(P=0.69)$.

The serum level of vitamin B12 was categorized into normal, deficient, and borderline for regression analysis. Univariate logistic regression analysis with delirium as the dependent variable and serum cobalamin level as the independent variable showed that there was no association between serum cobalamin level and delirium. Multivariate logistic regression analysis showed the same result (Table 2).

\section{Serum level of folic acid and outcomes}

The mean \pm SD serum level of folic acid was $12.39 \pm 6.32$ $\mathrm{ng} / \mathrm{mL}$. Folic acid deficiency with a serum level of lower than $2 \mathrm{ng} / \mathrm{mL}$ was seen in $6 \%(\mathrm{n}=18)$ of patients. The mean \pm SD serum level of folic acid was $10.77 \pm 5.39 \mathrm{ng} / \mathrm{mL}$ in delirium patients and $12.86 \pm 6.51 \mathrm{ng} / \mathrm{mL}$ in non-delirium patients, a difference not statistically significant $(P=0.008)$. The median length of stay in the ICU was $3(2-3)$ and $2(2-4)$ in patients 
Table I Baseline characteristics of patients based on delirium and non-delirium of subjects

\begin{tabular}{|c|c|c|c|c|}
\hline Variable & $\begin{array}{l}\text { Total } \\
(n=296)\end{array}$ & $\begin{array}{l}\text { Delirium } \\
(n=68)\end{array}$ & $\begin{array}{l}\text { Non-delirium } \\
(n=228)\end{array}$ & $P$-value \\
\hline Age (years) & $62(54-66)$ & $64(59-73)$ & $61(53-66)$ & $<0.00 I^{* *}$ \\
\hline Gender, n (\%) & & & & $0.92 * * *$ \\
\hline Female & $64(22)$ & $16(23)$ & $48(2 I)$ & \\
\hline Male & $232(78)$ & $52(77)$ & $180(79)$ & \\
\hline Education level, n (\%) & & & & $0.007 * * *$ \\
\hline Illiterate & $104(35)$ & $38(56)$ & $66(29)$ & \\
\hline Less than diploma & $136(46)$ & $24(35)$ & II2 (49) & \\
\hline Diploma & $40(14)$ & $0(0)$ & $40(18)$ & \\
\hline College education & $16(5)$ & $6(9)$ & $10(4)$ & \\
\hline NYHA classification, n (\%) & & & & $0.23 * * *$ \\
\hline Class II & $236(80)$ & $50(73)$ & $186(82)$ & \\
\hline Class III & $38(13)$ & $8(12)$ & $30(13)$ & \\
\hline Class IV & $22(7)$ & $10(15)$ & $12(5)$ & \\
\hline Type of surgery, n (\%) & & & & $0.50 * * *$ \\
\hline SVD & $20(7)$ & $6(9)$ & $14(6)$ & \\
\hline 2VD & $56(19)$ & $10(15)$ & $46(20)$ & \\
\hline 3VD & $208(70)$ & $50(73)$ & $158(69)$ & \\
\hline CABG+ valvular & $12(4)$ & $2(3)$ & $10(5)$ & \\
\hline Hemoglobin, ${ }^{a}(g / d L)$ & $13.70 \pm 1.83$ & $13.39 \pm 1.66$ & $13.80 \pm 1.88$ & $0.25^{*}$ \\
\hline \multicolumn{5}{|l|}{ Comorbidities } \\
\hline Diabetes mellitus, $\mathrm{n}(\%)$ & $132(45)$ & $30(44)$ & $102(45)$ & $0.65 * * *$ \\
\hline Hypertension, n (\%) & $150(5 \mathrm{I})$ & $42(62)$ & $108(47)$ & $0.04 * * *$ \\
\hline Hyperlipidemia n (\%) & $104(35)$ & $18(26)$ & $86(38)$ & $0.02 * * *$ \\
\hline Smoker, n (\%) & $82(28)$ & $14(2 \mid)$ & $68(30)$ & $0.34 * * *$ \\
\hline Addiction, n (\%) & $54(18)$ & $14(21)$ & $40(17)$ & $0.90 * * *$ \\
\hline Eye problem, n (\%) & $20(7)$ & $10(15)$ & $10(4)$ & $0.001 * * *$ \\
\hline Ear/language barrier, n (\%) & $26(9)$ & $8(12)$ & $18(8)$ & $0.29 * * *$ \\
\hline Alcohol use & 0 & 0 & 0 & 1 \\
\hline CPB duration (h:min) & $4: 3(4-4: 47)$ & $4: 3(3: 82-5: 07)$ & $4: 2(4: 0-4: 45)$ & $0.33 * *$ \\
\hline Post-surgery sodium, mg/dL & $140.76 \pm 12.04$ & $|42.9| \pm 2.9$ & $140.12 \pm 13.56$ & $0.005^{*}$ \\
\hline Post-surgery potassium, $\mathrm{mg} / \mathrm{dL}$ & $4.5(4.2-4.7)$ & $4.5(4.3-4.7)$ & $4.5(4.2-4.7)$ & $0.48 * *$ \\
\hline Post-surgery creatinine, $\mathrm{mg} / \mathrm{dL}$ & $1.20 \pm 0.32$ & $1.34 \pm 0.42$ & $1.16 \pm 0.27$ & $0.005^{*}$ \\
\hline Poor mobilization, n (\%) & $72(24)$ & $12(18)$ & $60(26)$ & $0.60 * * *$ \\
\hline Poor sleep, n (\%) & $48(16)$ & $8(12)$ & $40(17)$ & $0.54 * * *$ \\
\hline LOS ICU, days & $2(2-4)$ & $4(2-5)$ & $2(2-3)$ & $0.00 * *$ \\
\hline LOS hospital, days & $6(5-7)$ & $7(6-9)$ & $6(5-7)$ & $0.00 * *$ \\
\hline Acute infection, $\mathrm{n}(\%)$ & $6(2)$ & $2(3)$ & $4(2)$ & $0.45 * * *$ \\
\hline
\end{tabular}

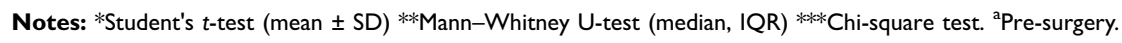

Abbreviations: NYHA, New York Heart Association Heart Function; SVD, single vessel disease; CABG, coronary artery bypass grafting; CPB, cardiopulmonary bypass; $2 \mathrm{VD}$, two vessel disease; 3VD, three vessel disease; LOS, length of stay; ICU, intensive care unit.

with folic acid deficiency and without folic acid deficiency, respectively; as is observed, these differences are not significant $(P=0.54)$.

The univariate logistic regression analysis with delirium as the dependent variable and categorized serum folic acid as the independent variable demonstrated that there was no relationship between the serum folic acid level and delirium.
Multivariate logistic regression analysis showed the same result (Table 2).

\section{Serum level of homocysteine and outcomes}

The median serum level of homocysteine was 17.55 (IQR, 14.2-23.1) $\mu \mathrm{mol} / \mathrm{L}$. Homocysteine serum level 
Table 2 Logistic regression analysis for delirium

\begin{tabular}{|c|c|c|c|c|c|c|}
\hline & OR & $95 \% \mathrm{Cl}$ & $P$-value & Adjusted OR* & $95 \% \mathrm{Cl}$ & $P$-value \\
\hline \multicolumn{7}{|l|}{ Delirium } \\
\hline Age & 1.06 & $1.03-1.10$ & 0.001 & 1.03 & $1.00-1.06$ & 0.03 \\
\hline Sodium $^{\mathrm{a}}$ & 1.11 & $1.02-1.21$ & 0.008 & & & \\
\hline Creatinine $^{a}$ & 5.6 & $2.56-12.27$ & 0.001 & 2.66 & $1.07-6.60$ & 0.03 \\
\hline Hyperlipidemia & 1.94 & $1.08-3.48$ & 0.02 & & & \\
\hline Hypertension & 0.58 & $0.34-0.99$ & 0.04 & & & \\
\hline Vision impairment & 0.25 & $0.10-0.60$ & 0.001 & & & \\
\hline \multicolumn{7}{|l|}{ Cobalamin subgroups: } \\
\hline Borderline $(n=104)$ & 1.36 & $0.74-2.52$ & 0.31 & 1.40 & $0.7 I-2.70$ & 0.32 \\
\hline Deficiency $(n=86)$ & 0.54 & $0.25-1.15$ & 0.11 & 0.69 & $0.30-1.55$ & 0.37 \\
\hline Normal $(n=106)$ & Reference & & & & & \\
\hline \multicolumn{7}{|l|}{ Folic acid subgroups: } \\
\hline Deficiency $(n=18)$ & 0.84 & $0.27-2.60$ & 0.76 & 0.97 & $0.27-3.50$ & 0.98 \\
\hline Normal $(n=278)$ & Reference & & & & & \\
\hline \multicolumn{7}{|l|}{ Homocysteine subgroups: } \\
\hline Mild $(n=186)$ & 1.57 & $0.66-3.70$ & 0.30 & 1.02 & $0.45-2.43$ & 0.82 \\
\hline Intermediate $(n=14)$ & 0 & 0 & 0.99 & 0 & 0 & 0.99 \\
\hline Normal $(n=96)$ & Reference & & & & & \\
\hline
\end{tabular}

Notes: *Adjusted by age, impaired vision, hypertension, hyperlipidemia, postoperative serum sodium and creatinine. ${ }^{\text {PPost-surgery. }}$

was normal in $32 \%(n=96)$ of the total population. Hyperhomocysteinemia was moderate in $63 \%(n=186)$ and intermediate in $5 \%(\mathrm{n}=14)$ of the total population. The median serum level of homocysteine was 18.5 (IQR, 14.55-22.15) $\mu \mathrm{mol} / \mathrm{L}$ in delirium patients and 17.33 (IQR, 14.2-23.27) $\mu \mathrm{mol} / \mathrm{L}$ in non-delirium patients, with a difference not much significant $(P=0.09)$. The median length of stay in the ICU was not different between the three subgroups of homocysteine $(P=0.42)$.

The results of univariate logistic regression analysis with delirium as the dependent variable and three categories of serum homocysteine level as the independent variable showed that there was no association between serum homocysteine levels and delirium. Multivariate logistic regression analysis showed the same result (Table 2).

\section{Relationship between cobalamin, folate, and homocysteine serum levels}

There existed a negative correlation between serum cobalamin levels and serum homocysteine levels $(r=-0.272$, $P=0.000$ ), and between serum folate and serum homocysteine $(r=-0.287, P=0.000)$.

Hyperhomocysteinemia was detected in $72 \%(n=62)$ of patients with cobalamin deficiency and in $33 \%(n=6)$ of patients with folate deficiency, respectively. In the total population, four patients had hyperhomocysteinemia with cobalamin and folate deficiency.

As homocysteine, folate and cobalamin are related to one another, and low serum levels of folate and cobalamin lead to increased serum homocysteine, an interactive model was employed through adding new variables in the regression analysis. In this model, all three variables were allowed to interact with one another in all possible two-way and three-way interactions. The results of logistic regression analysis showed no correlation with delirium.

\section{Discussion}

In the present study, no relationship was observed between the preoperative levels of cobalamin, folate, and homocysteine and delirium after CABG surgery. Although the serum level of folate was lower and the homocysteine level was higher in delirium patients, the difference was not statistically significant.

Folate and cobalamin play major roles in one-carbon cycle, which is crucial to the metabolism of monoamine neurotransmitter, and the formation and repair of nucleic acid in the central nervous system. Since one of the causes of delirium is the loss of balance between monoaminergic (norepinephrine, serotonin, and dopamine) and cholinergic 
neurotransmitter systems, folate, and cobalamin deficiency can be contributing factors in this disorder. Cobalamin and folate deficiency lead to hyperhomocysteinemia which is associated with Alzheimer disease, increased risk of cardiovascular disease, vascular dementia, cognitive impairment, and cerebral and hippocampal atrophy, hence a risk factor for delirium. ${ }^{20,21}$

In contrast to our results, Sevuk et al, showed that cobalamin deficiency was able to augment the risk of delirium in patients undergoing $\mathrm{CABG}$ surgery. In this retrospective study, they compared 200 patients (with and without cobalamin deficiency) undergoing CABG surgery. The incidence of delirium was higher in patients with cobalamin deficiency, and cobalamin level was significantly lower in delirium patients. ${ }^{14}$ Comparing this study with ours, Sevuk et al, merely assessed older patients, in a retrospective design which is not as strong as a prospective study.

In a study conducted by Kwok et al, 30 older subjects with mild to moderate dementia and low serum cobalamin deficiency were supplemented with vitamin B12 for 16 weeks. Their results showed no change in the cognitive function, but delirium rating scale score was reduced in the subjects. $^{22}$ Comparing the foregoing study with ours, it becomes clear that cobalamin deficiency is related to chronic delirium and not to the acute occurrence of delirium observed after cardiac surgery.

The relationship between the level of folate and homocysteine and acute cognitive disorder (POD) has not been specified yet. Moretti et al, assessed the association between Alzheimer's dementia with serum folate and Homocysteine levels, where they showed that hyperhomocysteinemia and low levels of folate serum were correlated with dementia. In this cross-sectional study, 450 cases with Alzheimer's disease and subcortical vascular dementia were compared with a healthy old volunteer population. It was observed that low levels of folate and high levels of homocysteine were more pronounced in subcortical vascular dementia cases. $^{23}$

Delirium following cardiac surgery is an acute condition. Although the pathophysiology of POD is not completely understood, acute changes in the normal physiologic condition of patient induce delirium. Several hypotheses regarding the pathophysiologic mechanisms of delirium are introduced. Hypoxia and microemboli, which lead to Ischemic brain damage following conditions such as hypotension, hypoxia, previous brain lesions, and extracorporeal circulation, can induce delirium. Imbalance of neurotransmitters (cholinergic deficiency and dopaminergic excess) in conditions such as medication, metabolic disturbances, and history of neurologic or psychiatric conditions are other proposed mechanisms. Furthermore, inflammation in the brain following operation, sepsis, and distress are some mechanisms effective in this regard. $^{24}$ Although cobalamin and folic acid deficiencies play a role in the metabolism of monoamine neurotransmitters, their deficiencies seem to be related in chronic conditions of dementia and cognitive functions. It is of note that the relation of these deficiencies with cognitive dysfunction and dementia in elderly, depression, dementia, catatonia, and schizophrenia has been reported previously. ${ }^{9-12}$

We found a negative correlation between serum cobalamin, folate, and homocysteine. Homocysteine is a sulfurcontaining amino acid biosynthesized from methionine, an essential amino acid. Homocysteine is an intermediate product in the transfer of activated methyl groups from tetrahydrofolate to S-adenosylmethionine (the reversible remethylation pathway). This reaction is catalyzed by the methionine synthetase and requires vitamin B12 as a cofactor and 5-methylTHF from folate as a methyl donor. So a deficiency of either vitamin B12 or folate can lead to Hyperhomocysteinemia.

The present study had its limitations and strengths. The prospective design of our study is among its strength, and the fact that CAM-ICU was employed in delirium assessment, which is a strong method and a validated tool. ${ }^{25}$ In our study, important confounders such as age, certain comorbidities, and the level of several electrolytes such as potassium and sodium were adjusted. The observational design of the research is its main limitation, as we were not able to find the exact mechanism. In addition, due to the cohort and single center design of the study, the generalizability of the results is low.

\section{Acknowledgments}

We convey our gratitude to the Faculty of Medicine, Tehran Medical Sciences, Islamic Azad University, Tehran, Iran and the ICU staff of Shahid Lavasani Hospital.

\section{Author contributions}

All authors contributed equally to the conception and design of the research. AV, MB, and SAJ contributed to the acquisition, analysis, and interpretation of the data. ZVS and MVS contributed to the analysis and interpretation of the data. All authors drafted and critically revised the manuscript, agree to be fully accountable for ensuring the integrity and accuracy of the work, and read and approved the final manuscript. 


\section{Disclosure}

The authors report no conflicts of interest in this work.

\section{References}

1. Rengel KF, Pandharipande PP, Hughes CG. Postoperative delirium. La Presse Médicale. 2018;47:e53-e64. doi:10.1016/j. lpm.2018.03.012

2. Vasilevskis EE, Han JH, Hughes CG, Ely EW. Epidemiology and risk factors for delirium across hospital settings. Best Pract Res Clin Anaesthesiol. 2012;26:277-287. doi:10.1016/j.bpa.2012.07.003

3. Bettelli G, Neuner B. Postoperative delirium: a preventable complication in the elderly surgical patient. Monaldi Arch Chest Dis. 2017;87:842-846. doi:10.4081/monaldi.2017.842

4. Smulter N, Lingehall HC, Gustafson Y, Olofsson B, Engstrom KG. Delirium after cardiac surgery: incidence and risk factors. Interact Cardiovasc Thorac Surg. 2013;17:790-796. doi:10.1093/icvts/ivt323

5. Barbateskovic M, Larsen LK, Oxenboll-Collet M, Jakobsen JC, Perner A, Wetterslev J. Pharmacological interventions for delirium in intensive care patients: a protocol for an overview of reviews. Syst Rev. 2016;5:211-215. doi:10.1186/s13643-016-0391-5

6. Lee H, Ju J-W, Oh S-Y, Kim J, Jung CW, Ryu HG. Impact of timing and duration of postoperative delirium: a retrospective observational study. Surgery. 2018:pii: S0039-6060(18)30035-7. doi:10.1016/j. surg.2018.02.001. Epub ahead of print.

7. Pawlak R, Lester SE, Babatunde T. Prevalence of cobalamin deficiency among vegetarians assessed by serum vitamin B12: a review of literature. Eur J Clin Nutr. 2014;68:541-548. doi:10.1038/ejen.2014.46

8. Yan M, Peng D, Liu C, Huang C, Luo J. Serum high concentrations of homocysteine and low levels of folic acid and vitamin B 12 are significantly correlated with the categories of coronary artery diseases. Cardiovasc Dis. 2017;17:37-41. doi:10.1186/s12872-0170475-8

9. Penninx BWJH, Guralnik JM, Ferrucci L, Fried LP, Allen RH, Stabler SP. Vitamin B12 deficiency and depression in physically disabled older women: epidemiologic evidence from the women's health and aging study. Am $J$ Psychiatry. 2000;157:715-721. doi:10.1176/appi.ajp.157.5.715

10. Werder SF. Cobalamin deficiency, hyperhomocysteinemia, and dementia. Neuropsychiatr Dis Treat. 2010;6:159-195.

11. Berry N, Sagar R, Tripathi BM. Catatonia and other psychiatric symptoms with vitamin B12 deficiency. Acta Psychiatr Scand. 2003;108:156-159

12. Cao B, Wang DF, Xu MY, et al. Vitamin B12 and the risk of schizophrenia: a meta-analysis. Schizophr Res. 2016;172:216-217. doi:10.1016/j.schres.2016.01.050
13. Torio I, Bagney A, Caballero M, Jimenez-Arriero MA, RodriguezJimenez R. Psychosis due to vitamin B12 deficiency in an elderly patient. Rev Neurol. 2014;59:45-47.

14. Sevuk U, Baysal E, Ay N, et al. Relationship between cobalamin deficiency and delirium in elderly patients undergoing cardiac surgery. Neuropsychiatr Dis Treat. 2015;11:2033-2039. doi:10.2147/NDT.S87888

15. Malouf M, Grimley EJ, Areosa SA. Folic acid with or without vitamin B12 for cognition and dementia. Cochrane Database Syst Rev. 2003;4:Cd004514.

16. Khanna S, Kapoor P, Pillai KK, Vohora D. Homocysteine in neurological disease: a marker or a cause? CNS Neurol Disord Drug Targets. 2011;10:361-369. doi:10.2174/187152711794653797

17. Ranucci M, Ballotta A, Frigiola A, et al. Pre-operative homocysteine levels and morbidity and mortality following cardiac surgery. Eur Heart J. 2009;30:995-1004. doi:10.1093/eurheartj/ehp015

18. Hyperhomocysteinemia - Hematology and Oncology - Merck Manuals Professional Edition; 2017. Available from: merckmanuals. com. Accessed September 6, 2017.

19. Antony AC. Megaloblastic anemias. In: Hoffman R, Benz EJ, Shattil SJ, editors. Hematology: Basic Principles and Practice. $4^{\text {th }}$ ed. New York: Churchill Livingstone; 2005:519.

20. Hshieh TT, Fong TG, Marcantonio ER, Inouye SK. Cholinergic deficiency hypothesis in delirium: a synthesis of current evidence. J Gerontol A Biol Sci Med Sci. 2008;63:764-772. doi:10.1093/gerona/63.7.764

21. Trzepacz PT. Is there a final common neural pathway in delirium? Focus on acetylcholine and dopamine. Semin Clin Neuropsychiatry. 2000;5:132-148.

22. Kwok T, Lee J, Lam L, Woo J. Vitamin B(12) supplementation did not improve cognition but reduced delirium in demented patients with vitamin $\mathrm{B}(12)$ deficiency. Arch Gerontol Geriatr. 2008;46:273-282. doi:10.1016/j.archger.2007.05.001

23. Moretti R, Caruso P, Dal Ben M, Conti C, Gazzin S, Tiribelli C. Vitamin D, homocysteine, and folate in subcortical vascular dementia and Alzheimer dementia. Front Aging Neurosci. 2017;9:169-174. doi:10.3389/fnagi.2017.00169

24. Trabold B, Metterlein T. Postoperative delirium: risk factors, prevention, and treatment. J Cardiothorac Vasc Anesth. 2014;28:1352-1360. doi:10.1053/j.jvca.2014.03.017

25. Ely EW, Inouye SK, Bernard GR, et al. Delirium in mechanically ventilated patients: validity and reliability of the confusion assessment method for the intensive care unit (CAMICU). JAMA 2001;286:2703-2710.
Neuropsychiatric Disease and Treatment

\section{Publish your work in this journal}

Neuropsychiatric Disease and Treatment is an international, peerreviewed journal of clinical therapeutics and pharmacology focusing on concise rapid reporting of clinical or pre-clinical studies on a range of neuropsychiatric and neurological disorders. This journal is indexed on PubMed Central, the 'PsycINFO' database and CAS, and is the official journal of The International Neuropsychiatric Association (INA). The manuscript management system is completely online and includes a very quick and fair peer-review system, which is all easy to use. Visit http://www.dovepress.com/testimonials.php to read real quotes from published authors. 